\title{
A Stochastic Geometry Approach to Full-Duplex MIMO Relay Network
}

\author{
Mhd Nour Hindia ${ }^{\mathbb{D}},{ }^{1}$ Moubachir Madani Fadoul, ${ }^{1}$ \\ Tharek Abdul Rahman, ${ }^{1}$ and Iraj Sadegh Amiri $\circledast^{2,3}$ \\ ${ }^{1}$ Wireless Communication Centre, Universiti Teknologi Malaysia, Skudai, Johor Bahru, Malaysia \\ ${ }^{2}$ Computational Optics Research Group, Ton Duc Thang University, Ho Chi Minh City, Vietnam \\ ${ }^{3}$ Faculty of Applied Sciences, Ton Duc Thang University, Ho Chi Minh City, Vietnam \\ Correspondence should be addressed to Iraj Sadegh Amiri; irajsadeghamiri@tdt.edu.vn
}

Received 24 June 2017; Revised 26 August 2017; Accepted 10 September 2017; Published 3 January 2018

Academic Editor: Patrick Seeling

Copyright (c) 2018 Mhd Nour Hindia et al. This is an open access article distributed under the Creative Commons Attribution License, which permits unrestricted use, distribution, and reproduction in any medium, provided the original work is properly cited.

Cellular networks are extensively modeled by placing the base stations on a grid, with relays and destinations being placed deterministically. These networks are idealized for not considering the interferences when evaluating the coverage/outage and capacity. Realistic models that can overcome such limitation are desirable. Specifically, in a cellular downlink environment, the full-duplex (FD) relaying and destination are prone to interferences from unintended sources and relays. However, this paper considered two-hop cellular network in which the mobile nodes aid the sources by relaying the signal to the dead zone. Further, we model the locations of the sources, relays, and destination nodes as a point process on the plane and analyze the performance of two different hops in the downlink. Then, we obtain the success probability and the ergodic capacity of the two-hop MIMO relay scheme, accounting for the interference from all other adjacent cells. We deploy stochastic geometry and point process theory to rigorously analyze the two-hop scheme with/without interference cancellation. These attained expressions are amenable to numerical evaluation and are corroborated by simulation results.

\section{Introduction}

Currently, the demand for the high capacity and low latency is dramatically increasing, corresponding to advancements in communication devices such as smartphones. It is expected that the wireless traffic volume of these communication devices will have a 1000-fold increase over the next decade which will be driven by the expected billions of connected devices by 2020 to access and share data anywhere and anytime [1]. The bottom line is that the technology has to move to higher frequency bands of millimeter wave to adapt the $5 \mathrm{G}$ future mobile communications, where a very large bandwidth in frequency band is available. With rapid increase in the number of connected devices, some challenges appear such as the capacity shortage, cost, and cochannel, and intercell interference especially at high dense network is rapidly increased [2]. Hence, the fifth-generation (5G) wireless systems should be able to support the ultradense networks to adopt the exponential growth in mobile users and high data demand. To satisfy the quality of service (QoS), it is expected that the $5 \mathrm{G}$ network deployment will be much denser compared to that of $4 \mathrm{G}$. However, as the network density increases, the interference will degrade the system's performance especially at cell edges (dead zone). One of these efficient solutions that allow the $5 \mathrm{G}$ network to meet its QoS requirements is to use relay to improve the ultradense network performance (capacity enhancement and coverage extension). Cooperative communication is an alternative way to achieve spatial diversity and multiplexing gain through Multiple-Input Multiple-Output (MIMO). MIMO attracted a lot of attention due to its potential for interference mitigation and capacity increase. FD relaying equipped with MIMO is able to perform spatial self-interference suppression [25]. The FD relaying looks to be an alternative solution to satisfy the high capacity demands of these wireless systems. The FD communication has attracted considerable attention 
of many researchers and it is expected to be integrated into the $5 \mathrm{G}$ wireless systems. Most used cooperative protocols are the amplify-and-forward (AF) and the decode-and-forward (DF); the DF cooperative protocols can operate in either a half-duplex (HD) or full-duplex (FD) mode. Multiplexing loss occurred when implementing the HD mode in the DF protocol; this is attributed to the fact that, in the first time/frequency slot, the relay has to wait for the source's message and then forward the message in the next time slot to the destination. However, FD cooperative protocols can overcome the HD spectral loss via simultaneous transmission from source to relay and from relay to destination; this enables frequency reuse, higher throughput, and lower transmission delay [6]. However, there are two main issues hardening the implementation of FD system in cellular network: (1) the self-interference, that is, the relay's signal leakage from its transmit and receive antennas, and (2) the fact that the simultaneous transmission creates intercell interference [7].

However, cellular networks are usually modeled by placing the sources on a grid (with a regular shape) or arranging them on a line or circle as in the Wyner model with the relay and destination being either deterministically or randomly placed across the network to determine the signalto-interference-and-noise ratio (SINR). The resulting SINR is complex and depends on multiple random variables. Hence, this fails to account for the randomness in the cellular network distribution [8] and the intercell interference [9]. Such models, however, are highly idealized and not tractable; hence, complex system level simulation is used to evaluate the outage/success probability and ergodic capacity. In order to reduce the dependence on simulations, the closed-form SINR was derived using stochastic geometry [10].

Since characterizing the SINR by the grid model and Wyner model is obviously not practicable, recently stochastic geometry has emerged as a powerful tool to model and quantify the capacity, interference, and success probability in cellular networks that are verified to be approximate to the actual networks [11]. The use of the Poisson point processes (PPP) model simplified the analysis and provided insight into the operation of the network in the form of scaling laws. Base station, relay, and destination parameters (e.g., path-loss exponent and transmit power) become the sign of the node in the PPP. Recently, under homogeneity condition, it was shown that the source positions are agnostic to the radio propagation; this makes the received power at the relay from any population of sources as if it generated from PPP, distributed sources $[12,13]$. This justifies the modeling assumption of PPP sources and allows computing some metrics performance such as the success probability and Ergodic capacity [14, 15], while [16] derived an upper bound for the success probability. Obtaining full diversity order using distributed space-time codes is detailed in $[17,18]$, but a distributed space-time code requires precise signaling and very tight coordination among the relays, which increases the complexity and overhead in the system. This paper is motivated by the benefit of MIMO two-hop system, such as performance benefits and reducing implementation complexity.

Researchers have dealt with the interference in many different ways. For instance, an active method known as analog cancellation, to cancel the interfering signal at the receiving antenna, utilizes additional radio frequency (RF) chains; and there is another active cancellation method known as digital cancellation, where the RSI is removed in the base-band level after the analog-to-digital converter [19]; another simple passive method is known as antenna separation, where the RSI is attenuated due to the path-loss between the transmitting and receiving antennas on the FD node. Further, reducing the FD interference using directional antennas is analyzed in [20]. References [21, 22] quantify the impact of self-interference of a heterogeneous network consisting of FD and HD nodes. They conclude that the capacity can be maximized by operating all nodes in either HD or FD compared to their mixtures, whereas [23] considers a single-cell setting and $[22,24]$ consider multicell setting. A cellular system comprising an FD source and HD destination has been illustrated in which the throughput gain is analyzed via extensive simulation [25]. In [26], the numbers of base station antennas and users antennas are increasing with a fixed ratio, while the capacity grows by the number of users and SNR. In this paper, however, we use a stochastic geometry tool to characterize the randomly distributed performance of FD MIMO relay nodes and derive bounds for the probability of successful transmission and ergodic capacity with/without interference cancellation. Finally, numerical results corroborate the theoretical findings with baseline scheme.

Next, in Section 2, the network system model is described. The SINR characterization is derived in Section 2.3; the success probability and the ergodic capacity are given in Section 2.4 and Section 2.5, respectively. Section 4 depicts the numerical results, and Section 5 offers the conclusion.

Throughout this paper, boldface lowercase letters (e.g., $\mathbf{x})$ represent vectors, and boldface uppercase letters (e.g., $\mathbf{X}$ ) represent matrices. $\|\cdot\|_{F}$ is the Frobenius norm. $\{\cdot\}^{\mathrm{H}}$ stands for conjugate transpose, and $\mathbb{E}[\cdot]$ denotes the expectation operator, while $|\bullet|$ denotes the magnitude and the trace of a matrix is denoted by $\operatorname{tr}\{\cdot\} ; \operatorname{det}(\cdot)$ is the determinant; $\operatorname{diag}\left(x_{1}, \ldots, x_{n}\right)$ is the diagonal matrix with diagonal components $x_{1}, \ldots, x_{n}$; $\mathbf{I}_{N \times N}$ is the $N$-by- $N$ identity matrix; $\mathbf{X}_{M \times N}$ is the $M$-by- $N$ zero matrix.

\section{System Model}

2.1. System Setup. In a downlink relay-assisted cellular network, consider a multiple independent FD dual-hop relaying system. The $j$ th source $S_{j}$ with message $\mathbf{x}_{S_{j}}$ equipped with $M_{s}$ antennas communicates with $N_{d}$ destinations $D_{j}$ receiving antennas through relay set $R_{j}$, with messages $\mathbf{x}_{R_{j}}$, equipped with $M_{r}$ and $N_{r}$ transmitting and receiving antennas, respectively, as in Figure 1. The relay uses DF protocol; hence, $R_{j}$ decodes the message from $S_{j}$ and then reencodes the message before sending it to $D_{j}$. The relays are equipped with FD capabilities, where the reception and transmission of relay signal happen simultaneously. The main challenge is that relay's transmitted signal is coupled with its receiver chain, causing relay self-interference (RSI) via $\mathbf{H}_{R_{j}}$ channel and source causes interference to other relay known as sourcerelay interference $(\mathrm{SRI})$ via $\mathbf{H}_{S_{i} R_{j}}$ channel in addition to 

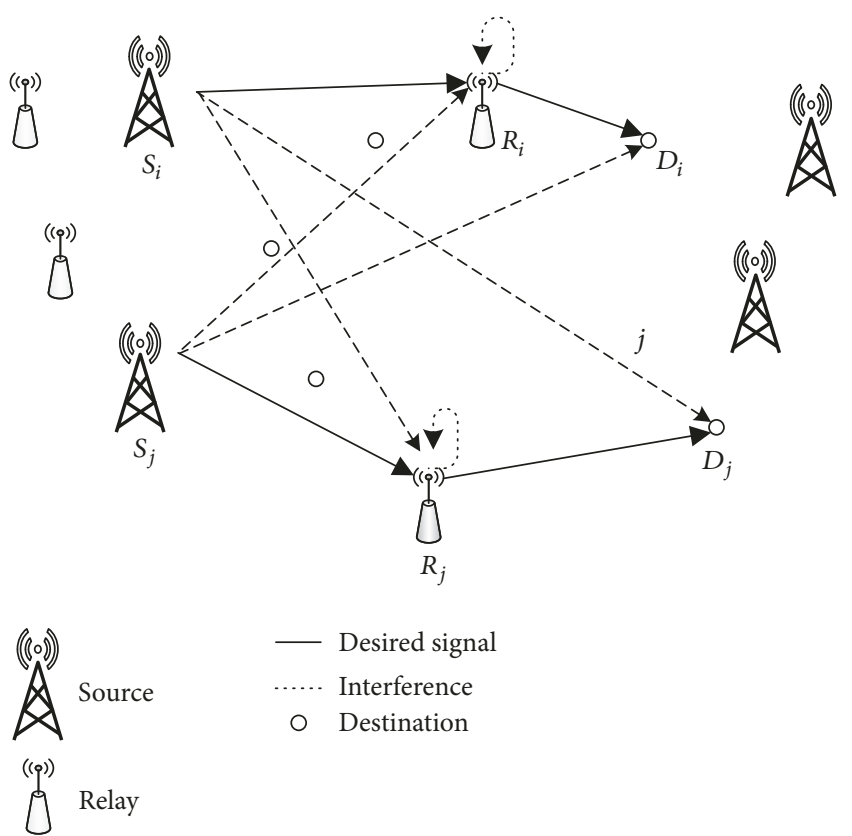

Figure 1: System model depicting cellular network, in which the source $(S)$ is having $M_{s}$ transmitting antennas, willing to communicate with $N_{d}$ receiving antennas, at the destination $(D)$, with the help of relay $(R)$, equipped with $M_{r}$ and $N_{r}$ transmitting and receiving antennas, respectively. The solid lines show desired signal, and the dashed lines show possible interferences such as RSI, IRI, SDI, and SRI.

the interference from the sources to the destinations causing source-destination interference (SDI) via $\mathbf{H}_{S_{i} D_{j}}$. Space division multiplexing is applied so that the two hops are separated.

The locations of sources, relays, and destinations are assumed to be stationary independently marked PPP with intensity $\lambda_{S}, \lambda_{R}$, and $\lambda_{D}$ on $\mathbb{R}^{2} \times \mathbb{R}^{2} \times \mathbb{R}^{2}$, respectively. In order to investigate the performance of a random access wireless network, consider a classical receiver located at the origin. Conditioning on the node's event lying at the origin does not affect the statistics of the rest of the process as an outcome from Palm probabilities of a Poisson process. Furthermore, the statistics of received signal at the intended receiver are observable by any receiver due to the stationarity of Poisson process [10].

2.2. Channel Model. The downlink cellular network is comprised of sources $S_{i}$ and $S_{j}$, relays $R_{i}$ and $R_{j}$, and destinations $D_{i}$ and $D_{j}$. Previous studies on cellular networks assumed that the sources and relays are positioned regularly as grid model. However, in practice, this is not true and there are some random characteristics. We applied homogeneous PPP to the spatial distribution of the sources and relays to remedy the model as in $[11,15]$. Each destination is served by the nearest relay or destination. This means that the cell area of each source or relay constitutes a Voronoi tessellation [27] as in Figure 2, whereas, in grid model in Figure 3, the sources are located on the centers of hexagonal model, while the relays and destinations are distributed in each cell uniformly. This

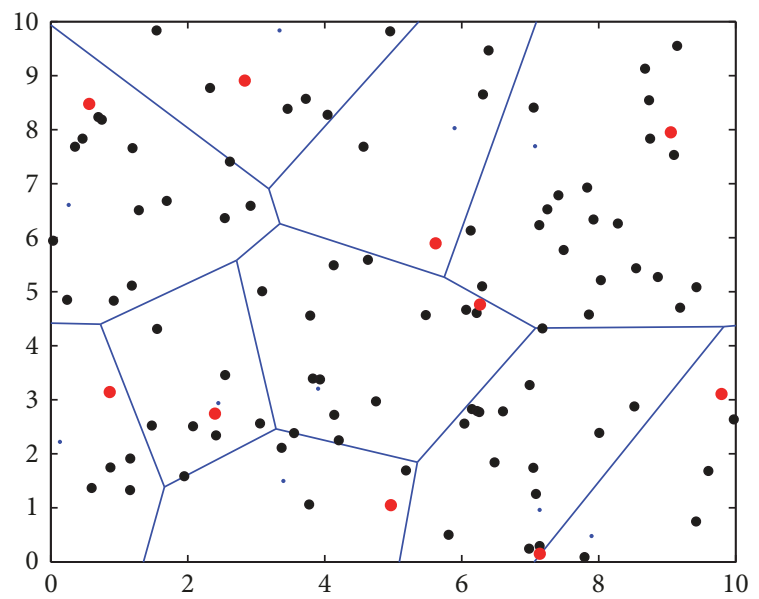

FIgURE 2: The cellular network topology, in which each Voronoi cell is the coverage area of a source and relay distributed as PPP; the cell area of each source and relay forms a Voronoi tessellation, where the red circle represents source, blue dot represents relay, and the black circle represents the destination.

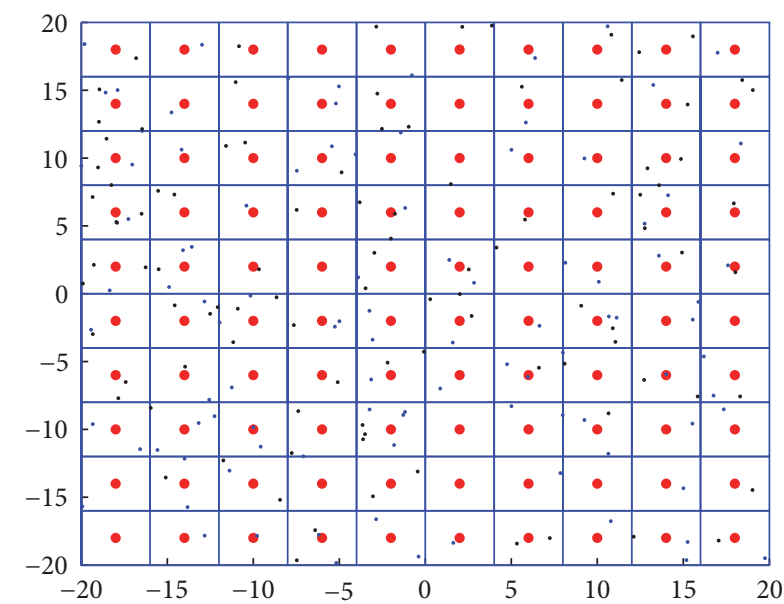

FIGURE 3: The grid cellular network topology, in which each Voronoi cell is the coverage area of a source distributed as PPP, where the red circle represents sources, blue dot represents relay, and the black circle represents the destination.

model does not lead to a tractable model; thus, it is evaluated via Monte Carlo simulations. When a destination attempts to access the sources, it chooses to connect directly or through relay in the Voronoi cell. Let the source $S_{j}$ transmit the message $\mathbf{x}_{S_{j}}$ to the $j$ th relay $R_{j} ; R_{j}$ receives and transmits simultaneously on the same frequency causing RSI through the channel $\mathbf{H}_{R_{j}}$ if $R_{j} \in \Phi$ for the same $j$ th relay $R_{j}$ and receives IRI through the channel $\mathbf{H}_{R_{i} R_{j}}$ if $R_{i} R_{j} \in \Phi$ from the other $i$ th relay $R_{i}$, in addition to interference from other source $S_{i} . \mathbf{x}_{S_{j}} \in \mathbb{C}^{M_{\mathrm{s}} \times 1}$ and $\mathbf{x}_{R_{j}} \in \mathbb{C}^{M_{\mathbf{r}} \times 1}$ are transmitted signals from $S_{j}$ and $R_{j}$ nodes respectively.

The propagation through two-hop wireless channel is subject to path-loss attenuation modeled as $\widetilde{R}^{-\alpha}$ and $\widehat{R}^{-\alpha} \forall x \in \Phi$ for distances $\widetilde{R}$ and $\widehat{R}_{j}$, respectively, with path-loss exponent $\alpha>2$. The sources and relays transmit with power $\widetilde{\rho}$ and power $\hat{\rho}$. For such channel, the sources cover large areas, 
while relays cover small areas with separation distances $\widetilde{R}_{j}$ and $\widehat{R}_{j}\left(\widetilde{R}_{i} \gg \widehat{R}_{j}\right)$ given $\tilde{\rho} \mathbf{H}_{S_{j} R_{j}} \widetilde{R}_{j}^{-\alpha}$ if $S_{j} R_{j} \in \Phi$ and $\hat{\rho} \mathbf{H}_{R_{j} D_{j}} \widehat{R}_{j}^{-\alpha}$ if $R_{j} D_{j} \in \Phi$. The interfering relays constitute the marked process $\Phi=\left\{\left(X_{i}, \mathbf{H}_{R_{i} R_{j}}, \mathbf{H}_{R_{j}}\right)\right\}$, with $X_{i}$ denoting the location of the transmitting relay node. Hence, the relay receives interference power from itself, $\hat{\rho} \mathbf{H}_{R_{j}}$, and from the $i$ th interfering relay and source as $\hat{\rho} \mathbf{H}_{R_{i} R_{j}}\left|X_{i}\right|^{-\alpha}$ and $\widetilde{\rho} \mathbf{H}_{S_{i} R_{j}}\left|X_{i}\right|^{-\alpha}$, respectively. The channels $\mathbf{H}_{S_{j} R_{j}} \in \mathbb{C}^{N_{r} \times M_{s}}, \mathbf{H}_{R_{j}} \in \mathbb{C}^{N_{\mathbf{r}} \times M_{r}}$, $\mathbf{H}_{R_{i} R_{j}} \in \mathbb{C}^{N_{r} \times M_{r}}, \mathbf{H}_{R_{j} D_{j}} \in \mathbb{C}^{N_{d} \times M_{r}}$, and $\mathbf{H}_{S_{j} D_{i}} \in \mathbb{C}^{M_{s} \times N_{d}}$ are the channel gain matrices from source-to-relay, relay itself, interrelay, relay-to-destination, and source-to-destination, respectively. The power constraints on transmit signals are $\mathbb{E}\left\{\left|\mathbf{x}_{S_{j}}\right|^{2}\right\}=1, \mathbb{E}\left\{\left|\mathbf{x}_{R_{j}}\right|^{2}\right\}=1$, and $\mathbb{E}\left\{\left|\mathbf{x}_{R_{j} R_{j}}\right|^{2}\right\}=1 . \mathbf{y}_{R_{j}} \in \mathbb{C}^{N_{r} \times 1}$ and $\mathbf{y}_{D_{j}} \in \mathbb{C}^{N_{d} \times 1}$ are received signals at $R_{j}$ and $D_{j}$ nodes. All channels experience Rayleigh fading with parameter $\mu \sim \operatorname{Exp}(\mu)$. The entries of each matrix are independent and identically distributed (i.i.d.) complex Gaussian variables with zero mean and variance $\boldsymbol{\sigma}^{2} \cdot \mathbf{n}_{R}$ and $\mathbf{n}_{D}$ are independent circularly symmetric complex Gaussian noise vectors with probability distributions $\mathcal{N}(0, \mathbf{I})$ and $\mathscr{N}\left(0, \mathbf{I}_{D}\right)$, respectively, and are uncorrelated to $\mathbf{x}_{S}$ and $\mathbf{x}_{R}$.

2.3. SINR Characterization. In this section, we formulate the signal-to-interference-plus-noise ratio (SINR), which is needed in the next section to analyze the probability of successful transmission. In doing so, we study the SINR of the SR hop and the SINR of the RD hop.

2.3.1. SR Hop. Consider the $j$ th source node $S_{j}$ located at the origin and, according to the stationarity of $\Phi$ and building on Slivnyak's theorem [10, Ch. 8.5], we hence express the statistics of relay-received signal $l\left(R_{j}, x\right)=\left|X_{x}\right|^{-\alpha}$, with $\left|X_{x}\right|$ being the distance of $x$ from the origin. Therefore the received signal at the $j$ th relay node contains the desired signal plus interference and noise ratio, RSI, and SRI, as given as follows:

$$
\begin{aligned}
& \mathbf{y}_{R_{j}} \triangleq \underbrace{\left(\sqrt{\tilde{\boldsymbol{\rho}}} \widetilde{R}_{j}{ }^{-\boldsymbol{\alpha} / \mathbf{2}} \mathbf{H}_{S_{j} R_{j}} \mathbf{x}_{S_{j}}+\mathbf{n}_{R_{j}}\right)}_{\text {desired signal plus noise }}+\underbrace{\sqrt{\widehat{\boldsymbol{\rho}}} \mathbf{H}_{R_{j}} \widehat{\mathbf{x}}_{S_{j}}}_{\text {RSI }} \\
& +\underbrace{\sqrt{\tilde{\rho}} \widetilde{R}_{j}^{-\alpha / 2} \mathbf{H}_{S_{i} R_{j}} \widehat{\mathbf{x}}_{S_{i}}}_{\text {SRI }} .
\end{aligned}
$$

The resulting SINR at the relay is obtained as

$$
\mathrm{SINR}_{R_{j}} \triangleq \frac{\widetilde{\rho} \widetilde{R}_{j}^{-\alpha}\left|\mathbf{H}_{S_{j} R_{j}}\right|^{2}}{I_{R_{j}}+\sigma_{R_{j}}^{2}}
$$

where $I_{R_{j}}$ is the overall interference (RSI and SRI) at jth relay; that is,

$$
I_{R_{j}} \triangleq \sum_{\{i, j\} \in \Phi}\left(\widetilde{\rho} \widetilde{R}_{j}^{-\alpha}\left|\mathbf{H}_{S_{i} R_{j}}\right|^{2}\right)+\left(\hat{\rho}\left|\mathbf{H}_{R_{j}}\right|^{2}\right) .
$$

For simplicity, we assume that this hop is interference-limited and, therefore, $I_{R_{j}} \gg \sigma_{R_{j}}^{2}$.
2.3.2. $R D$ Hop. Consider the $j$ th relay node $S_{j}$ located at the origin and, according to the stationarity of $\Phi$ and building on Slivnyak's theorem, we express $l\left(D_{j}, x\right)=\left|X_{x}\right|^{-\alpha}$, with $\left|X_{x}\right|$ being the distance of $x$ from the origin. Therefore the received signal at the $j$ th destination node contains the desired signal plus interference and noise ratio, as shown as follows:

$$
\mathbf{y}_{D_{j}} \triangleq \underbrace{\sqrt{\widehat{\boldsymbol{\rho}}} \widehat{R}_{j}^{-\boldsymbol{\alpha} / 2} \mathbf{H}_{R_{j} D_{j}} \widehat{\mathbf{x}}_{S_{j}}+\mathbf{n}_{D_{j}}}_{\text {desired signal plus noise }}+\underbrace{\sqrt{\tilde{\boldsymbol{\rho}} \widetilde{R}_{i}^{-\boldsymbol{\alpha} / 2} \mathbf{H}_{S_{i} D_{j}} \widehat{\mathbf{x}}_{D_{j}}}}_{\text {SDI }}
$$

The resulting SINR at the destination is

$$
\mathrm{SINR}_{D_{j}} \triangleq \frac{\widehat{\rho} \widehat{R}_{j}^{-\alpha}\left|\mathbf{H}_{R_{j} D_{j}}\right|^{2}}{I_{D_{j}}+\sigma_{D_{j}}^{2}}
$$

where $I_{D_{j}}$ captures the interference from other source to destination for the RD hop:

$$
I_{D_{j}} \triangleq \sum_{\{i, j\} \in \Phi}\left(\widetilde{\rho} \widetilde{R}_{j}^{-\alpha}\left|\mathbf{H}_{S_{i} D_{j}}\right|^{2}\right)
$$

For simplicity, we assume that this hop also is interferencelimited and therefore $I_{D_{j}} \gg \sigma_{D_{j}}^{2}$. Furthermore, the mean link distance of $I_{S_{i} R_{j}}$ and $I_{R_{i} R_{j}}$ is $\mathbb{E}\left[I_{S_{i} R_{j}}\right]=\widetilde{\rho} \widetilde{R}^{-\alpha}$ and $\mathbb{E}\left[I_{R_{i} R_{j}}\right]=$ $\hat{\rho} \bar{R}_{j}{ }^{\alpha}$, respectively, such that $\mathbb{E}\left[I_{S_{i} R_{j}}\right] \ggg \mathbb{E}\left[I_{R_{i} R_{j}}\right]$.

2.4. Success Probability. One of our performance measures is the success probability, which, defined as the success probability of message transmission from the source to destination over the two hops, is given by a joint complementary cumulative distribution function (CCDF) of $\operatorname{SINR}_{R_{j}}$ and $\operatorname{SINR}_{D_{j}}$. We formulate it by $P_{\text {suc }} \triangleq \mathbb{P}\left(\operatorname{SINR}_{R_{j}} \geq \theta_{R}, \operatorname{SINR}_{D_{j}} \geq \theta_{D}\right)$, where $\theta$ is the given SINR threshold. Due to independent sampling of a point process, there is no correlation between the two hops.

$$
P_{\text {suc }}=P_{\text {suc }}^{(\mathrm{SR})} P_{\text {suc }}^{(\mathrm{RD})},
$$

where $P_{\text {suc }}^{(\mathrm{SR})} \triangleq \mathbb{P}\left(\operatorname{SINR}_{R_{j}} \geq \theta_{R}\right)$ and $P_{\text {suc }}^{(\mathrm{RD})} \triangleq \mathbb{P}\left(\operatorname{SINR}_{D_{j}} \geq \theta_{D}\right)$.

\subsubsection{SR Hop. Successful SR hop transmission occurs if}

$$
\frac{\widetilde{\rho} \widetilde{R}_{j}^{-\alpha}\left|\mathbf{H}_{S_{j} R_{j}}\right|^{2}}{I_{R_{j}}} \geq \theta_{R}
$$

the given threshold $\theta$ satisfied a target SINR; the probability of successful transmission for a relay is 


$$
\begin{aligned}
\mathbb{P} & \left(\operatorname{SINR}_{R_{j}} \geq \theta\right)=\mathbb{P}\left(\frac{\widetilde{\rho} \widetilde{R}_{j}^{-\alpha}\left|\mathbf{H}_{S_{j} R_{j}}\right|^{2}}{I_{R_{j}}} \geq \theta_{R}\right) \\
& =\mathbb{P}\left(\left|\mathbf{H}_{S_{j} R_{j}}\right|^{2} \geq \theta_{R} \widetilde{\rho}^{-1} \widetilde{R}_{j}^{\alpha} I_{R_{j}}\right) \\
& =\int_{0}^{\infty} \mathbb{P}\left(\left|\mathbf{H}_{S_{j} R_{j}}\right|^{2} \geq s \theta_{R} \widetilde{\rho}^{-1} \widetilde{R}_{j}^{\alpha}\right) f_{I_{R_{J}}}(s) d s \\
& =\int_{0}^{\infty} F_{\mathrm{SR}}^{c}\left(s \theta \widetilde{\rho}^{-1} \widetilde{R}_{j}^{\alpha}\right) f_{I_{R_{J}}}(s) d s,
\end{aligned}
$$

where (9) is obtained by conditioning $s$ and its complementary cumulative distribution function is denoted by $F^{c}(\cdot)$. Next, we provide the success probability analysis of the SR hop.

Theorem 1. Let the interfering relays transmitters form a Poisson process of intensity $\lambda_{R}$ around relay receivers. The success probability of the SR hop is

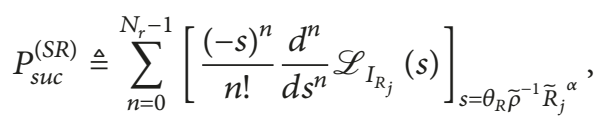

where $\mathscr{L}_{I_{R_{j}}}$ is the Laplace transform of the interferences $I_{R_{j}}$.

$$
\begin{aligned}
\mathscr{L}_{I_{R_{j}}}(s) & \triangleq \frac{1}{(1+s \tilde{\rho})} \exp \left(-\lambda_{S} \Upsilon(s)\right) \\
\Upsilon(s) & \triangleq \int_{0}^{\infty}\left(2 \pi-\frac{1}{1+s \widetilde{\rho} r^{-\alpha}} \Psi(s, r)\right) r d r
\end{aligned}
$$

with

$$
\Psi(s, r) \triangleq \int_{0}^{2 \pi} \frac{d \varphi}{1+s \widetilde{\rho}\left(\widetilde{R}_{j}^{2}+r^{2}+2 \widetilde{R}_{j} r \cos \varphi\right)^{-\alpha / 2}} .
$$

Proof. Refer to Appendix B.

The result in Theorem 1 provides a fundamental limit on the SR hop and its performance in an interference-limited scenario.

\subsection{2. $R D$ Hop. Successful RD hop transmission occurs if}

$$
\begin{aligned}
\mathbb{P}\left(\operatorname{SINR}_{D_{j}} \geq \theta_{D}\right) & =\mathbb{P}\left(\frac{\hat{\rho} \widehat{R}_{j}^{-\alpha}\left|\mathbf{H}_{R_{j} D_{j}}\right|^{2}}{I_{D_{j}}} \geq \theta_{D}\right) \\
& =\mathbb{P}\left(\left|\mathbf{H}_{R_{j} D_{j}}\right|^{2} \geq \theta_{D} \widehat{\rho}^{-1} \widehat{R}_{j}^{\alpha} I_{D_{j}}\right) .
\end{aligned}
$$

Next, we provide the success probability analysis of the $\mathrm{RD}$ hop.

Theorem 2. Let the relays transmission form a Poisson process of intensity $\lambda_{D}$ around destinations. The success probability of the RD hop is

$$
P_{s u c}^{(R D)} \triangleq \sum_{n=0}^{N_{d}-1}\left[\frac{(-s)^{n}}{n !} \frac{d^{n}}{d s^{n}} \mathscr{L}_{I_{D_{j}}}(s)\right]_{s=\theta_{D} \widehat{P}^{-1} \widehat{R}_{j}^{\alpha}},
$$

where $\mathscr{L}_{I_{D_{j}}}$ is the Laplace transform of the interference $I_{D_{j}}$ :

$$
\mathscr{L}_{I_{D_{j}}}(s) \triangleq \Psi\left(s, \widehat{R}_{j}\right) \exp \left(-\lambda_{D} \Upsilon(s)\right),
$$

where $\Psi(s, r)$ and $\Upsilon(s)$ are already defined in (13) and (12), respectively.

Proof. Refer to Appendix C.

Corollary 3. The Laplace transform in (16) is bounded by $\mathscr{L}_{I_{D_{j}}}(s) \in\left[\mathscr{L}_{I_{D_{j}}}^{\min }(s), \mathscr{L}_{I_{D_{j}}}^{\max }(s)\right]$, where

$$
\begin{aligned}
& \mathscr{L}_{I_{D_{j}}}^{(\min )}(s) \triangleq \frac{1}{1+s \hat{\rho}\left(\widetilde{R}_{j}-\widehat{R}_{j}\right)^{-1}} \exp \left(-\lambda \Upsilon^{(\max )}(s)\right), \\
& \mathscr{L}_{I_{D_{j}}^{(\max )}}(s) \triangleq \frac{1}{1+s \widehat{\rho}\left(\widetilde{R}_{j}+\widehat{R}_{j}\right)^{-1}} \exp \left(-\lambda \Upsilon^{(\min )}(s)\right) \text {, }
\end{aligned}
$$

with $\Upsilon^{(\min )}(s)$ and $\Upsilon^{(\max )}(s)$ already being given in [21, 28] as follows:

$$
\begin{aligned}
& \Upsilon^{(\max )}(s) \triangleq 2(\tilde{\rho}+\hat{\rho}) \frac{\pi^{2} s^{2 / \alpha}}{\alpha \sin (2 \pi / \alpha)}, \\
& \Upsilon^{(\text {min })}(s) \triangleq\left(1+\frac{2}{\alpha}\right)(\hat{\rho}+\widetilde{\rho}) \frac{\pi^{2} s^{2 / \alpha}}{\alpha \sin (2 \pi / \alpha)} .
\end{aligned}
$$

This corollary will help us in proving the interference bound of success probability $\mathrm{P}_{\text {suc }}^{\mathrm{RD}}$ of RD hop; this can be done by substituting $\mathscr{L}_{I_{D_{j}}}(s) \in\left[\mathscr{L}_{I_{D_{j}}}^{\min }(s), \mathscr{L}_{I_{D_{j}}}^{\max }\right]$ in (15).

2.5. Ergodic Capacity. The other performance measure is the ergodic capacity; the goal of FD relay is to increase the network capacity; for the relay served by source, the following theorems give the outage probability (CDF of SINR) and the SR hop capacity.

Theorem 4. The probability that $\operatorname{SINR}_{R_{j}}$ falls below a given target level $\theta_{R}$ is known as outage probability, which is the $C D F$ of SINR $R_{R_{j}}$ of the SR hop denoted by $Z_{j}\left(\theta_{R}\right)=\mathbb{P}\left\{S I N R_{R_{j}} \leq \theta_{R}\right\}$ and given by

$$
Z_{R_{j}}\left(\theta_{R}\right)=1-\exp \left(-2 \pi \lambda_{S}\right),
$$

and similarly for the RD hop

$$
Z_{R_{j}}\left(\theta_{D}\right)=1-\exp \left(-2 \pi \lambda_{R}\right) .
$$

Hence, the SR hop capacity can be shown as

$$
C_{S_{j} R_{j}} \triangleq \sum_{j=1}^{N_{r}} \log \left(1+\theta_{R}\right) P_{\text {suc }}^{(S R)}
$$

and the RD hop capacity is

$$
C_{R_{j} D_{j}} \triangleq \sum_{j=1}^{N_{d}} \log \left(1+\theta_{D}\right) P_{s u c}^{(R D)} .
$$


The total channel capacity is denoted by

$$
C=\min \left(\left(C_{S_{j} R_{j}}\right),\left(C_{R_{j} D_{j}}\right)\right) .
$$

Proof. Refer to Appendix A.

For the single-antenna case, the following corollary shows that the FD relay outperforms the HD relay in terms of capacity.

Corollary 5. Consider the SR hop where relay and destination nodes are having only single antenna $N_{r}=N_{d}=1$. Letting $s=\theta_{R} \widetilde{\rho}^{-1} \widetilde{R}^{\alpha}$, the FD relay capacity is lower-bounded by

$$
\begin{aligned}
& C_{S_{j} R_{j}} \\
& \quad \triangleq \frac{2}{(1+s \tilde{\rho})} \exp \left(-\lambda_{S} \Upsilon^{(\max )}(s)\right) \sum_{j=1}^{N_{r}} \log \left(1+\theta_{R}\right) ;
\end{aligned}
$$

on the other hand, the HD relay capacity is upper-bounded by

$$
C_{S_{j} R_{j}}^{H D} \triangleq \exp \left(-\lambda_{S} \tilde{\rho} \frac{2 \pi^{2} s^{2 / \alpha}}{\alpha \sin (2 \pi / \alpha)}\right) \sum_{j=1}^{N_{r}} \log \left(1+\theta_{R}\right) ;
$$

therefore, $C_{S_{j} R_{j}} \geq C_{S_{j} R_{j}}^{H D}$ as long as the following condition holds:

$$
\lambda_{S} \leq \frac{\alpha \sin (2 \pi / \alpha)}{2 \pi^{2} s^{2 / \alpha}} \log _{2}\left(\frac{2}{1+s}\right) .
$$

Proof. The proof is obtained by combining Corollary 3 and Theorem 2.

It is evident that increasing the density $\lambda_{S}$ beyond the threshold does not compensate for the additional RSI, even if we use twice the FD capacity; this is due to FD simultaneous transmission and reception. Hence, the HD relay is optimal in this case.

2.6. Minimum Throughput Gain. We demonstrate the feasibility of FD relay over its counterpart, the HD relay, for the SR hop capacity only, but similar approach can be applied to the $\mathrm{RD}$ hop as well since the total capacity follows (23). We define a performance metric called the minimum throughput gain, which shows the worst case FD relay performance over HD relay in terms of capacity as follows:

$$
\mathrm{TG}^{\min } \triangleq \frac{C_{S_{j} R_{j}}}{C_{S_{j} R_{j}}^{\mathrm{HD}}}
$$

when TG $>1$, the FD relay outperforms the HD relay.

\section{Interference Cancellation}

This section considers the interference cancellation at the MIMO receiving nodes, that is, for SR hop at the relay and for $\mathrm{RD}$ hop at the destination. We present a low complexity spatial interference cancellation scheme known as partial zero forcing (PZF) [29, 30]. During the SR hop and RD hop, PZF can be applied at the relay and destination to cancel $K \leq N_{r}-1$ and $L \leq N_{d}-1$ interferences, respectively, while using the remaining degree of freedom to boost the intended received signal.
3.1. SR Hop: Canceling RSI and SRI. It is beneficial to increase the capacity by canceling the interference (RSI and SRI) from nearby nodes in an interference-limited scenario. Let us denote the points of $\{i, j\} \in \Phi$, in an increasing order from the source, that is, $\left\{\widetilde{R}_{i} \leq \widetilde{R}_{i+1}\right\}_{i=1}^{\infty}$; the relay applies PZF to cancel its $K$ interference. From (3), the overall interference can be rewritten as

$$
I_{R_{j}}(K) \triangleq \sum_{\substack{j \in \Phi \\ j>K}}\left(\widetilde{\rho} \widetilde{R}_{j}^{-\alpha}\left|\mathbf{H}_{S_{i} D_{j}}\right|^{2}\right)+\left(\hat{\rho}\left|\mathbf{H}_{R_{j}}\right|^{2}\right) .
$$

For the SR hop, with PZF at the relay, the success probability is given next.

Theorem 6. The success probability of the SR hop with PZF at the relay is

$$
P_{s u c}^{(S R)} \triangleq \sum_{n=0}^{N_{r}-K-1}\left[\frac{(-s)^{n}}{n !} \frac{d^{n}}{d s^{n}} \mathscr{L}_{I_{R_{j}}}(k)(s)\right]_{s=\theta_{R} \tilde{\rho}^{-1} \widetilde{R}_{j}{ }^{\alpha}},
$$

where $\mathscr{L}_{I_{R_{j}}(K)}(s)$ is the Laplace transform of the interferences:

$$
\mathscr{L}_{I_{R_{j}}(K)}(s) \triangleq \frac{1}{(1+s \tilde{\rho})} \mathbb{E}_{\Phi}\left[\prod_{\substack{j \in \Phi \\ j \leq K}} \frac{1}{1+s \tilde{\rho} r^{-\alpha}}\right] .
$$

Proof. See Appendix A and follow similar steps to those in [30].

3.2. RD Hop: Canceling SDI. It is beneficial to increase the capacity by canceling the interference (SDI) from nearby nodes in an interference limited scenario. Let us denote the points of $\{i, j\} \in \Phi$, in an increasing order from the source, that is, $\left\{\widehat{R}_{i} \leq \widehat{R}_{i+1}\right\}_{i=1}^{\infty}$; the destination applies PZF to cancel its $L$ interference. From (6), the overall interference can be rewritten as

$$
I_{D_{j}}(L) \triangleq \sum_{\substack{j \in \Phi \\ j>L}}\left(\hat{\rho} \widehat{R}_{j}^{-\alpha}\left|\mathbf{H}_{S_{i} D_{j}}\right|^{2}\right)
$$

and its corresponding success probability is given below.

Theorem 7. The success probability of the RD hop with PZF at the destination is

$$
P_{s u c}^{(R D)} \triangleq \sum_{n=0}^{N_{d}-L-1}\left[\frac{(-s)^{n}}{n !} \frac{d^{n}}{d s^{n}} \mathscr{L}_{I_{D_{j}}(L)}(s)\right]_{s=\theta_{D} \hat{\rho}^{-1} \widehat{R}_{j}^{\alpha}},
$$

where $\mathscr{L}_{I_{D_{j}}(L)}(s)$ is the Laplace transform of the interferences:

$$
\mathscr{L}_{I_{D_{j}}(L)}(s) \triangleq \frac{1}{(1+s \hat{\rho})} \mathbb{E}_{\Phi}\left[\prod_{\substack{j \in \Phi \\ j \leq L}} \frac{1}{1+s \hat{\rho} r^{-\alpha}}\right] .
$$

Proof. See Appendix A and follow similar steps as those in [30]. 


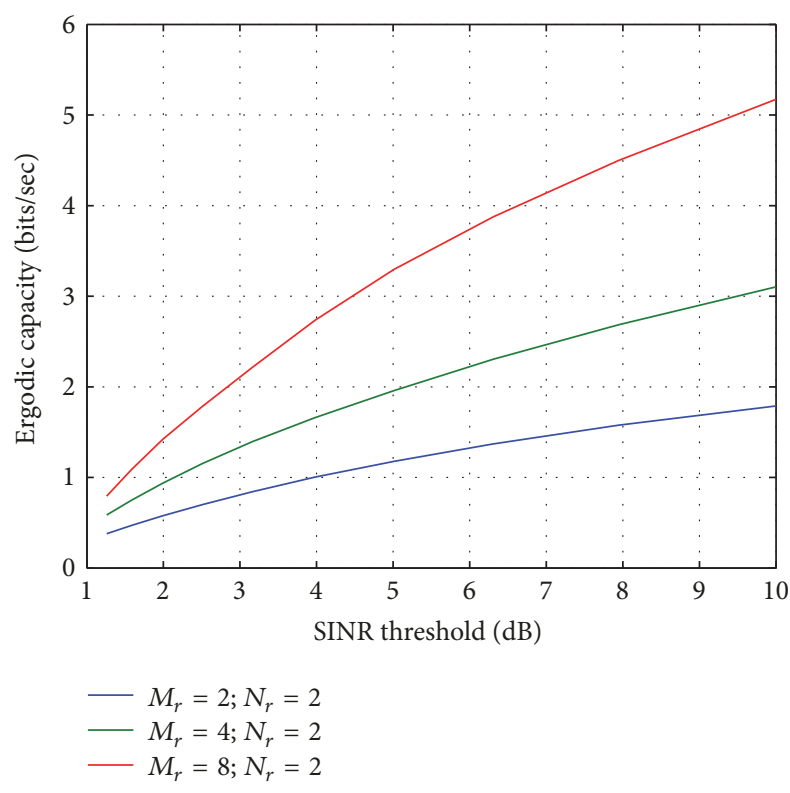

FIGURE 4: Ergodic capacity versus SINR threshold for different relay and destination transmitted and received antennas, respectively.

\section{Numerical Results}

Several Monte Carlo simulations are presented to validate the proposed scheme and averaged over 10000 number of channel realizations. Rayleigh fading assumption results in a convenient coverage probability form in terms of the Laplace transform of the interference, which is easier to characterize than the probability density function of the interference [10]. The sources and relays are transmitting with $\tilde{\rho}=1 \mathrm{~W}$ and $\widehat{\rho}=0.5 \mathrm{~W}$ with their corresponding distances $\widetilde{R}=5 \mathrm{~m}$ and $\widehat{R}=0.5 \mathrm{~m}$, respectively. The SINR threshold is $\theta=0 \mathrm{~dB}$ and the path-loss exponent is $\alpha=2.3$, with 100 destination nodes, 100 source nodes, and 100 relay nodes, with $N=8$ interfering source and relay nodes. We assume that, in this openaccess case, destinations (users) are able to connect to any source (base station). Open access requirements often are not satisfied, particularly deploying Wi-Fi in the multi-RAT (radio access technology) femtocells network [31].

Figure 4 shows the ergodic capacity in (23) of $M_{s}=N_{r}=$ 2, $M_{r}=N_{d}=2$ and $M_{s}=N_{r}=2, M_{r}=N_{d}=4$ and $M_{s}=$ $N_{r}=2, M_{r}=N_{d}=8$, antenna configurations. From (21) and (22) it is obvious that increasing the number of antennas employed at the relay and destination adds a positive term to the ergodic capacity; however, the total capacity is limited by the weakest hop (23). It can be observed that the ergodic capacity scales linearly with the number of antennas.

Figure 5 shows that the success probability in (7) increases with increasing the number of transmitting antennas at the source across the whole SINR. Deploying multiple antennas at the relay allows reducing the effect of interference, hence obtaining a substantial SINR gain. Figure 6 indicates that the success probability decreases with an increase in the SINR threshold. It compares the traditional grid model constituted by a Voronoi tessellation (see Figure 3 ) to the random PPP source model. Considering $N=8$ and $N=24$, it can be

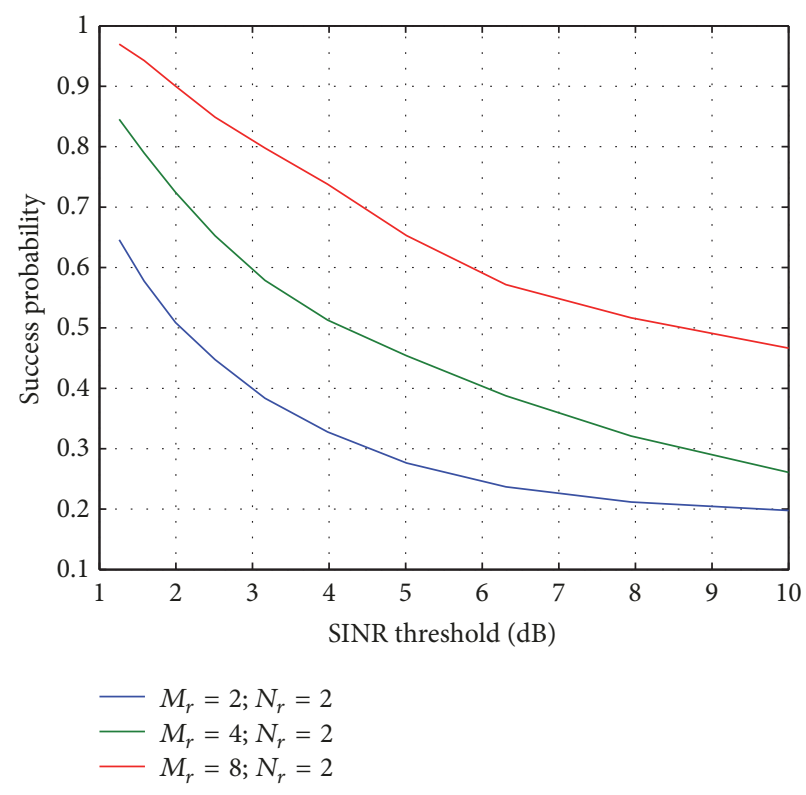

FIGURE 5: Success probability versus SINR threshold.

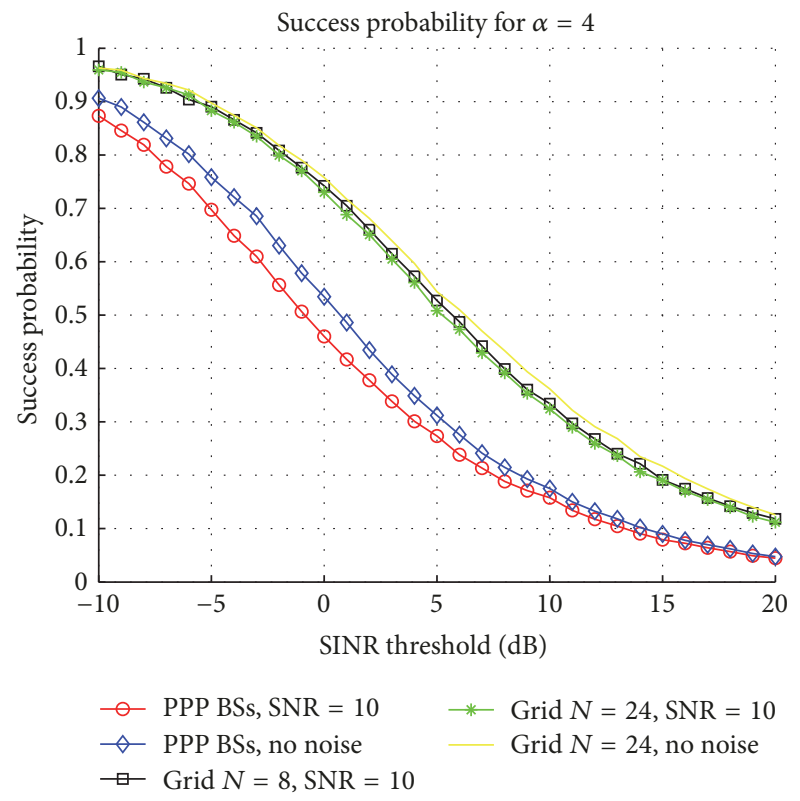

FIGURE 6: Success probability comparison between our proposed PPP source model and grid model with $N=8$ and $N=24$ and $\alpha=4$. No noise assumption is quite correct, and it can be observed that the case with $N=24$ is slightly lower than $N=8$.

observed that the case of $N=8$ is almost overlapped with $N=24$, for $\alpha=4$. As expected, the grid model provides high coverage area across the whole SINR. A small gap has been observed when considering the SNR $=10$ and SNR $\rightarrow \infty$, due to the noise effect in dense cellular networks, which are known to be interference-limited. This validates the assumption that the noise can be ignored in interferencelimited scenario.

Figure 7 presents a crucial issue in FD relay communications by evaluating the performance of multiple receive 


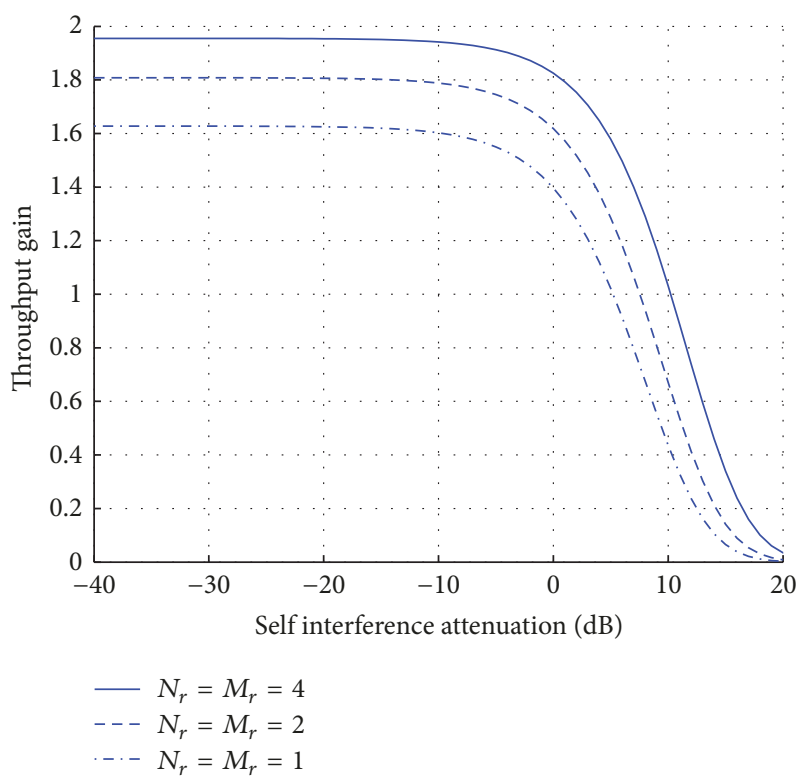

FIGURE 7: Comparison between FD and HD relay in the SR hop: minimum throughput gain for different values of self-interference attenuation with $\lambda=10^{-3}$ and $\theta=0 \mathrm{~dB}$.

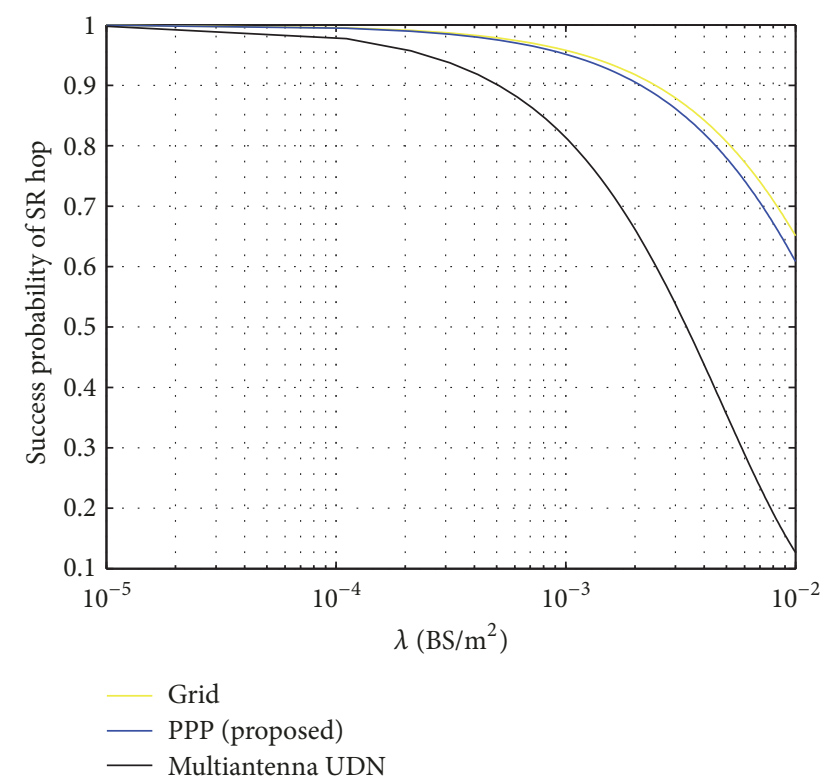

FIgURE 8: Success probability of the SR hop with interference cancellation versus the density $\lambda$ with $M_{s}=N_{r}=M_{r}=2$ and SINR threshold $\theta=0 \mathrm{~dB}$ for different schemes.

antennas in canceling the self-interference. The capacity gain is plotted against the self-interference attenuation with $\theta=$ $0 \mathrm{~dB}$ and $\lambda=10^{-3}$. We observed that $\mathrm{TG}^{\mathrm{min}}>1$, even for moderate self-interference attenuation values $\leq 4, \leq 8$, and $\leq 10 \mathrm{dBs}$ for $N_{r}=M_{r}=1, N_{r}=M_{r}=2$, and $N_{r}=M_{r}=4$, respectively.

For the SR hop, the relays are equipped with two receive antennas $N_{r}=2$ and deploy PZF to cancel either the RSI or the SRI. Figure 8 shows the success probability of the SR hop against the density $\lambda$ and compares it with the multiantenna UDN model [30] and the grid model. Note that the grid model is best suited for higher densities, whereas our proposed model performs slightly better than the multiantenna UDN model, due to the use of both antennas at source and relay for array gain to exploit the MIMO capability in boosting the intended received signal.

\section{Conclusion}

In a MIMO relay cellular network, RSI, SRI, and SDI will likely be the main obstacle reducing the capacity. This paper, however, developed a tractable model to analyze the success probability and ergodic capacity expressions for the random nodes, using tools from stochastic geometry. It is more realistic than the grid-based models and requires an extensive comparison with real base station deployments to validate its accuracy. We showed the effect of PZF in mitigating the interferences including the self-interference to demonstrate the feasibility of FD technology even for moderate values of self-interference attenuation. Future work probably uses this model extensively due to the interference modeling from neighboring nodes.

\section{Appendix}

\section{A.}

Assume that the typical source is located at the origin o, since the relay always chooses its nearest source to access; the cumulative density function (CDF) of $\widetilde{R}_{j}$ is obtained by

$$
\begin{gathered}
\mathbb{P}\left\{\widetilde{R}_{j}>\bar{R}_{j}\right\}=1-\mathbb{P}\left\{\text { no source closer than } \bar{R}_{j}\right\} \\
1-e^{-\lambda_{S} \pi \bar{R}_{j}^{2}} .
\end{gathered}
$$

Then, the probability density function (PDF) of $\widetilde{R}_{j}$ is $f\left(\widetilde{R_{j}}\right)=$ $e^{-\lambda_{S} \pi \widetilde{R}_{j}^{2}} 2 \pi \lambda_{S} \widetilde{R}_{j}$ as obtained SINR in (2). The CDF of SINR regarding $I_{R_{j}}$ is given by

$$
\begin{aligned}
& Z_{R_{j}}(\theta)=1-\mathbb{E}\left[\mathbb{P}\left\{\operatorname{SINR}_{R_{j}}>\theta_{R}\right\}\right] \\
& =1-\int_{0}^{\infty} 2 \pi \lambda_{S} \widetilde{R}_{j} e^{-\pi \lambda_{S} \widetilde{R}_{j}^{2} \mathbb{P}}\left\{\frac{\tilde{\rho} \widetilde{R}_{j}^{-\alpha}\left|\mathbf{H}_{S_{j} R_{j}}\right|^{2}}{I_{R_{j}}+\sigma_{j}^{2}}\right. \\
& \left.>\theta_{R}\right\} d \widetilde{R}_{j} \\
& =1-\int_{0}^{\infty} 2 \pi \lambda_{S} \widetilde{R}_{j} e^{-\pi \lambda_{S} \widetilde{R}_{j}^{2} \mathbb{P}}\left\{\left|\mathbf{H}_{S_{j} R_{j}}\right|^{2}\right. \\
& \left.>\frac{\theta_{R} \widetilde{R}_{j}^{\alpha}}{\widetilde{\rho}}\left(I_{R_{j}}+\sigma_{R_{j}}^{2}\right)\right\} d \widetilde{R}_{j} \text {. }
\end{aligned}
$$


Using the fact that $\left|\mathbf{H}_{S_{j} R_{j}}\right|^{2}=\sum_{j=1}^{M_{s}}\left|h_{S_{j} R_{j}}\right|^{2}$ with $h \sim \exp (1)$ simplifies to

$$
\begin{aligned}
& =1-\int_{0}^{\infty} 2 \pi \lambda_{S} \widetilde{R}_{j} e^{-\pi \lambda_{S} \widetilde{R}_{j}^{2}} \mathbb{E}\left\{e^{-\left(\theta \widetilde{R}_{j}^{-\alpha} / \widetilde{\rho}\right)\left(I_{R_{j}}+\sigma_{R_{j}}^{2}\right)}\right\} d \widetilde{R}_{j} \\
& =1-\int_{0}^{\infty} 2 \pi \lambda_{S} \widetilde{R}_{j} e^{-\pi \lambda_{S} \widetilde{R}_{j}^{2}-\left(\theta_{R} \widetilde{R}_{j}^{-\alpha} \sigma_{R_{j}}^{2} / \widetilde{\rho}\right)} \mathscr{L}_{R_{j}}\left(\frac{\theta_{R} \widetilde{R}_{j}^{\alpha}}{\widetilde{\rho}}\right) d \widetilde{R}_{j} .
\end{aligned}
$$

Now, Let us evaluate the Laplace transform for the interference $\mathscr{L}_{R_{j}}(s)$ building on the fact that the typical relay is served by the nearest source with distance $\widetilde{R}_{j}$. Firstly, the Laplace transform of the interference of relay can be obtained by following the same steps as in [10, Page 125]:

$$
\mathscr{L}_{I_{R_{j}}}(s)=\mathbb{E}\left\{\exp \left(-s \frac{\hat{\rho}}{N_{r_{i}}} \sum_{i \in \Phi \backslash\{j\}}\left|\mathbf{H}_{R_{j}}\right|^{2}\right)\right\} .
$$

Using the fact that $\left|\mathbf{H}_{R_{j}}\right|^{2}=\sum_{j=1}^{M_{r}}\left|h_{R_{j}}\right|^{2}$ with $h \sim \exp (1)$ simplifies to

$$
\begin{aligned}
& =\mathbb{E}\left(\prod_{\{i\} \in \Phi \backslash\{j\}} \mathbb{E}_{g_{j}}\left\{\exp \left(-s \frac{\rho}{N_{r}} g_{j}\right)\right\}\right) \\
& =\mathbb{E}\left(\prod_{\{i\} \in \Phi \backslash\{j\}} \frac{1}{1+\left(s\left(\hat{\rho} / N_{r}\right)\right)}\right) .
\end{aligned}
$$

Applying the probability generating functional of PPP $\Phi$ with intensity $\lambda$ for function $v(x)$ [32]:

$$
\mathbb{E}\left[\Pi_{x \in \Phi} v(x)\right]=\exp \left(-\lambda \int_{\mathbb{R}^{2}}(1-v(x)) d x\right) .
$$

Applying the probability generating function to $\mathscr{L}_{I_{R_{j}}}$ yields

$$
\begin{aligned}
& =\exp \left(-2 \pi \lambda_{S} \int_{\widetilde{R}}^{\infty}\left(1-\frac{1}{1+(s x)}\right) x d x\right) \\
& \mathscr{L}_{I_{R_{j}}}=\exp \left(-2 \pi \lambda_{S} \int_{\widetilde{R}}^{\infty}\left(1-\frac{1}{1+\left(\left(\theta_{R} \widetilde{R}_{j}^{\alpha} / \widetilde{\rho}\right) x\right)}\right)\right. \\
& \cdot x d x) \\
& \mathscr{L}_{I_{D_{j}}}=\exp -\left(2 \pi \lambda_{R} \int_{\widehat{R}}^{\infty}\left(1-\frac{1}{1+\left(\left(\theta_{D} \widehat{R}_{j}^{\alpha} / \hat{\rho}\right) x\right)}\right)\right. \\
& \cdot x d x) .
\end{aligned}
$$

Plugging (A.8) into (A.3) and assuming that the distance between $\widetilde{R}_{j}$ and $\widehat{R}_{j}$ is fixed, (19) and (20) are proven.

\section{B.}

Let us define $f_{I_{R_{j}}}(t)=d \mathbb{P}\left(I_{R_{j}} \leq t\right)$ as the PDF of $I_{R_{j}}$. The integration of $f_{I_{R_{j}}}(t)$ using the CCDF $F_{\mathrm{SR}}^{c}(t)$ transforms it as

$$
g(s)=\int_{0}^{\infty} F_{\mathrm{SR}}^{c}(s t) f_{I_{R_{j}}}(t) d t .
$$

Note that the power of desired signal is distributed as $\left|\mathrm{H}_{S_{j} R_{j}}\right|^{2} \sim \chi_{2 N_{r}}^{2}$; the probability of success is given by

$$
\begin{aligned}
\mathbb{P} & \left(\operatorname{SINR}_{R_{j}} \geq \theta_{R}\right)=\mathbb{P}\left(\left|\mathbf{H}_{S_{j} R_{j}}\right|^{2} \geq \theta_{R} \widetilde{\rho}^{-1} \widetilde{R}^{\alpha} I_{R_{j}}\right) \\
& =\int_{0}^{\infty} F_{\mathrm{SR}}^{c}(s t) f_{I_{R_{j}}}(t) d t \\
& =\left.g(s)\right|_{s=\theta_{R} \widetilde{\rho}^{-1} \widetilde{R}^{\alpha} .}
\end{aligned}
$$

Assume that $F_{\mathrm{SR}}^{c}(t)=e^{-t}$ and, using Laplace transform, the success probability can be expressed using the transformation of $f_{I_{R_{j}}}(t)$ :

$$
\begin{aligned}
g(s) & =\int_{0}^{\infty} F_{\mathrm{SR}}^{c}(s t) f_{I_{R_{j}}}(t) d t=\mathscr{L}\left\{f_{I_{R_{j}}}(t)\right\}(s) \\
& =\mathscr{L}_{I_{R_{j}}}(s) .
\end{aligned}
$$

Finally, using the $\operatorname{CCDF} F_{\mathrm{SR}}^{c}(t)=\sum_{n} e^{-n t} \sum_{j} a_{n} t^{j}$, the transformation of $f_{I_{R_{j}}}(t)$ is given by

$$
\begin{aligned}
g(s) & =\int_{0}^{\infty} F_{\mathrm{SR}}^{c}(s t) f_{I_{R_{j}}}(t) d t \\
= & \int_{0}^{\infty}\left(\sum_{n} e^{-n s t} \sum_{j} a_{n j}(s t)^{j}\right) f_{I_{R_{j}}}(t) d t \\
= & \sum_{n} \sum_{j} a_{n j} s^{j}\left(\int_{0}^{\infty} e^{-n t} t^{j} f_{I_{R_{j}}}(t) d t\right) \\
= & \sum_{n} \sum_{j} a_{n j} s^{j} \mathscr{L}\left\{t^{j} f_{I_{R_{j}}}(t)\right\}(n s) \\
= & \sum_{n} \sum_{j}\left[a_{n j}(-s)^{j} \frac{d^{j}}{d(n s)^{j}} \mathscr{L}_{I_{R_{j}}}(n s)\right] \\
& =\sum_{n} \sum_{j}\left[a_{n j}\left(-\frac{s}{n}\right)^{j} \frac{d^{j}}{d(n s)^{j}} \mathscr{L}_{I_{R_{j}}}(n s)\right]_{n s=n \theta \tilde{\rho}^{-1} \widetilde{R}^{\alpha}},
\end{aligned}
$$

where (B.6) is obtained by using Laplace transform property $t^{n} f(t) \longleftrightarrow(-1)^{n}\left(d^{n} / d s^{n}\right) \mathscr{L}[f(t)](s)$. Apply the momentgenerating function of $x_{2}$ and Gamma distribution to [211, Theorem 1] to reach (11).

C.

The success probability of the RD hop is shown as follows:

$$
\mathbb{P}\left(\operatorname{SINR}_{D_{j}} \geq \theta_{D}\right)=\mathbb{P}\left(\left|\mathbf{H}_{R_{j} D_{j}}\right|^{2} \geq \theta_{D} \widehat{\rho}^{-1} \widehat{R}_{j}^{\alpha} I_{D_{j}}\right),
$$


where $I_{D_{j}}$ is defined in (6). The desired signal is distributed as $\left|\mathbf{H}_{R_{j} D_{j}}\right|^{2} \sim \chi_{2 M_{r}}^{2}$; the proof of (15) is similar to Theorem 1. On the other hand, again we use the moment-generating function of $\chi_{2}^{2}$ distribution to provide the Laplace transform of $I_{D_{j}}[10$, Page 125] and resort to

$$
\begin{aligned}
& \mathscr{L}_{I_{D_{j}}}(s) \\
& \quad=\mathbb{E}\left(\prod_{\{i, j\} \in \Phi} \mathbb{E}\left\{\exp \left(-s \frac{\hat{\rho}}{N_{d}} \widehat{R}^{-\alpha}\left|\mathbf{H}_{R_{j} D_{j}}\right|^{2}\right)\right\}\right) .
\end{aligned}
$$

Hence, we prove (16).

\section{Conflicts of Interest}

The authors declare that they have no conflicts of interest.

\section{References}

[1] C.-X. Wang, F. Haider, X. Gao et al., "Cellular architecture and key technologies for $5 \mathrm{G}$ wireless communication networks," IEEE Communications Magazine, vol. 52, no. 2, pp. 122-130, 2014.

[2] T. S. Rappaport, R. W. Heath Jr., R. C. Daniels, and J. N. Murdock, Millimeter Wave Wireless Communications, Pearson Education, London, UK, 2014.

[3] Y. Hua, "An overview of beamforming and power allocation for MIMO relays," in Proceedings of the IEEE Military Communications Conference (MILCOM '10), pp. 375-380, San Jose, Claif, USA, November 2010.

[4] T. Riihonen, S. Werner, and R. Wichman, "Residual selfinterference in full-duplex MIMO relays after null-space projection and cancellation," in Proceedings of the 44th Asilomar Conference on Signals, Systems and Computers (Asilomar 10), pp. 653-657, Pacific Grove, Claif, USA, November 2010.

[5] D. W. Bliss, P. A. Parker, and A. R. Margetts, "Simultaneous transmission and reception for improved wireless network performance," in Proceedings of the IEEE/SP 14th WorkShoP on Statistical Signal Processing (SSP '07), pp. 478-482, Madison, Wis, USA, August 2007.

[6] Z. Zhou and X. Zhang, "Directional antenna-based single channel full duplex," IET Communications, vol. 9, no. 16, pp. 1999-2006, 2015.

[7] G. C. Alexandropoulos, M. Kountouris, and I. Atzeni, "User scheduling and optimal power allocation for full-duplex cellular networks," in Proceedings of the 17th IEEE International Workshop on Signal Processing Advances in Wireless Communications (SPAWC '16), Edinburgh, UK, July 2016.

[8] J. Xu, J. Zhang, and J. G. Andrews, "On the accuracy of the wyner model in cellular networks," IEEE Transactions on Wireless Communications, vol. 10, no. 9, pp. 3098-3109, 2011.

[9] R. Bhagavatula and J. Heath, "Adaptive bit partitioning for multicell intercell interference nulling with delayed limited feedback," IEEE Transactions on Signal Processing, vol. 59, no. 8, pp. 3824-3836, 2011.

[10] M. Haenggi, Stochastic Geometry for Wireless Networks, Cambridge University Press, Cambridge, UK, 2012.

[11] J. G. Andrews, F. Baccelli, and R. K. Ganti, "A tractable approach to coverage and rate in cellular networks," IEEE Transactions on Communications, vol. 59, no. 11, pp. 3122-3134, 2011.
[12] B. Błaszczyszyn, M. K. Karray, and H. P. Keeler, "Wireless networks appear poissonian due to strong shadowing," IEEE Transactions on Wireless Communications, vol. 14, no. 8, pp. 4379-4390, 2015.

[13] H. P. Keeler, N. Ross, and A. Xia, "When do wireless network signals appear poisson?” https://arxiv.org/abs/1411.3757.

[14] H. S. Dhillon, R. K. Ganti, and J. G. Andrews, "A tractable framework for coverage and outage in heterogeneous cellular networks," in Proceedings of the Information Theory and Applications Workshop (ITA '11), pp. 361-366, La Jolla, Claif, USA, February 2011.

[15] H. S. Dhillon, R. K. Ganti, F. Baccelli, and J. G. Andrews, "Modeling and analysis of K-tier downlink heterogeneous cellular networks," IEEE Journal on Selected Areas in Communications, vol. 30, no. 3, pp. 550-560, 2012.

[16] H. S. Dhillon, M. Kountouris, and J. G. Andrews, "Downlink coverage probability in MIMO HetNets," in Proceedings of the 46th Asilomar Conference on Signals, Systems and Computers (ASILOMAR '120, pp. 683-687, Pacific Grove, Claif, USA, November 2012.

[17] J. N. Laneman, D. N. Tse, and G. . Wornell, "Cooperative diversity in wireless networks: efficient protocols and outage behavior," IEEE Transactions on Information Theory, vol. 50, no. 12, pp. 3062-3080, 2004.

[18] J. N. Laneman and G. W. Wornell, "Distributed space-time coded protocols for exploiting cooperative diversity in wireless networks," IEEE Transactions on Information Theory, vol. 49, no. 10, pp. 2415-2425, 2003.

[19] M. Duarte, C. Dick, and A. Sabharwal, "Experiment-driven characterization of full-duplex wireless systems," IEEE Transactions on Wireless Communications, vol. 11, no. 12, pp. 4296-4307, 2012.

[20] C. Psomas, M. Mohammadi, I. Krikidis, and H. A. Suraweera, "Impact of directionality on interference mitigation in fullduplex cellular networks," IEEE Transactions on Wireless Communications, vol. 16, no. 1, pp. 487-502, 2017.

[21] Z. Tong and M. Haenggi, "Throughput analysis for wireless networks with full-duplex radios," in Proceedings of the IEEE Wireless Communications and Networking Conference (WCNC '15), pp. 717-722, New Orleans, La, USA, March 2015.

[22] J. Lee and T. Q. S. Quek, "Hybrid full-/half-duplex system analysis in heterogeneous wireless networks," IEEE Transactions on Wireless Communications, vol. 14, no. 5, pp. 2883-2895, 2015.

[23] M. Mohammadi, H. A. Suraweera, Y. Cao, I. Krikidis, and C. Tellambura, "Full-duplex radio for uplink/downlink wireless access with spatially random nodes," IEEE Transactions on Communications, vol. 63, no. 12, pp. 5250-5266, 2015.

[24] D. Gesbert, S. Hanly, H. Huang, S. Shamai Shitz, O. Simeone, and W. Yu, "Multi-cell MIMO cooperative networks: a new look at interference," IEEE Journal on Selected Areas in Communications, vol. 28, no. 9, pp. 1380-1408, 2010.

[25] S. Goyal, P. Liu, S. Hua, and S. Panwar, "Analyzing a full-duplex cellular system," in Proceedings of the 47th Annual Conference on Information Sciences and Systems (CISS '13), pp. 1-6, IEEE, Baltimore, Md, USA, March 2013.

[26] H. Huang and S. Venkatesan, "Asymptotic downlink capacity of coordinated cellular networks," in Proceedings of the Conference Record of the Thirty-Eighth Asilomar Conference on Signals, Systems and Computers, pp. 850-855, Pacific Grove, Claif, USA, November 2004. 
[27] T. D. Novlan, H. S. Dhillon, and J. G. Andrews, "Analytical modeling of uplink cellular networks," IEEE Transactions on Wireless Communications, vol. 12, no. 6, pp. 2669-2679, 2013.

[28] Z. Gong and M. Haenggi, "Interference and outage in mobile random networks: expectation, distribution, and correlation," IEEE Transactions on Mobile Computing, vol. 13, no. 2, pp. 337349, 2014.

[29] N. Jindal, J. G. Andrews, and S. Weber, "Rethinking MIMO for wireless networks: Linear throughput increases with multiple receive antennas," in Proceedings of the IEEE International Conference on Communications (ICC '09), Dresden, Germany, June 2009.

[30] I. Atzeni and M. Kountouris, "Performance analysis of partial interference cancellation in multi-antenna UDNs," in Proceedings of the 50th Asilomar Conference on Signals, Systems and Computers (ACSSC '16), pp. 699-703, Pacific Grove, Claif, USA, November 2016.

[31] S. Singh, H. S. Dhillon, and J. G. Andrews, "Offloading in heterogeneous networks: modeling, analysis, and design insights," IEEE Transactions on Wireless Communications, vol. 12, no. 5, pp. 2484-2497, 2013.

[32] S. N. Chiu, D. Stoyan, W. S. Kendall, and J. Mecke, Stochastic Geometry and Its Applications, John Wiley \& Sons, Hoboken, NJ, USA, 2013. 


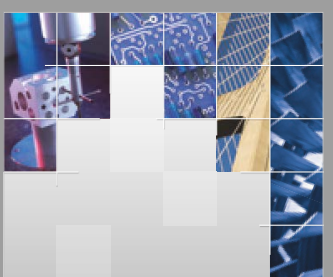

\section{Enfincering}
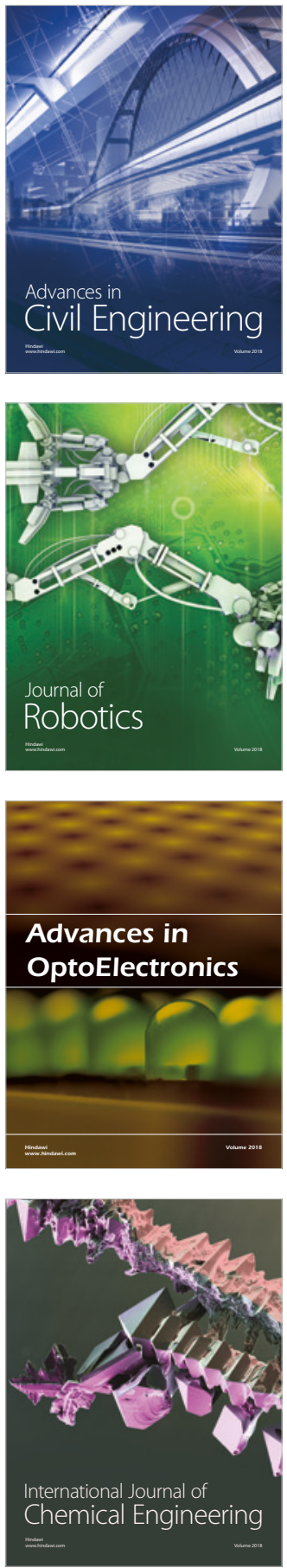

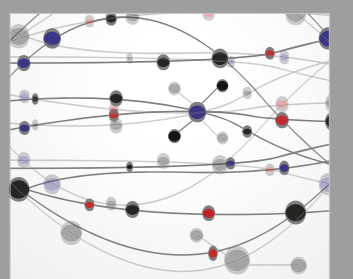

\section{Rotating \\ Machinery}

The Scientific World Journal

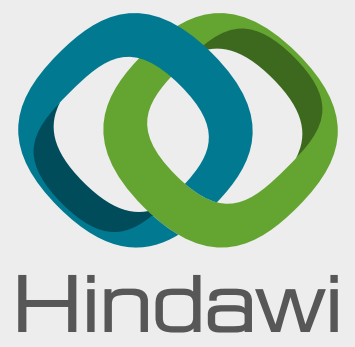

Submit your manuscripts at

www.hindawi.com
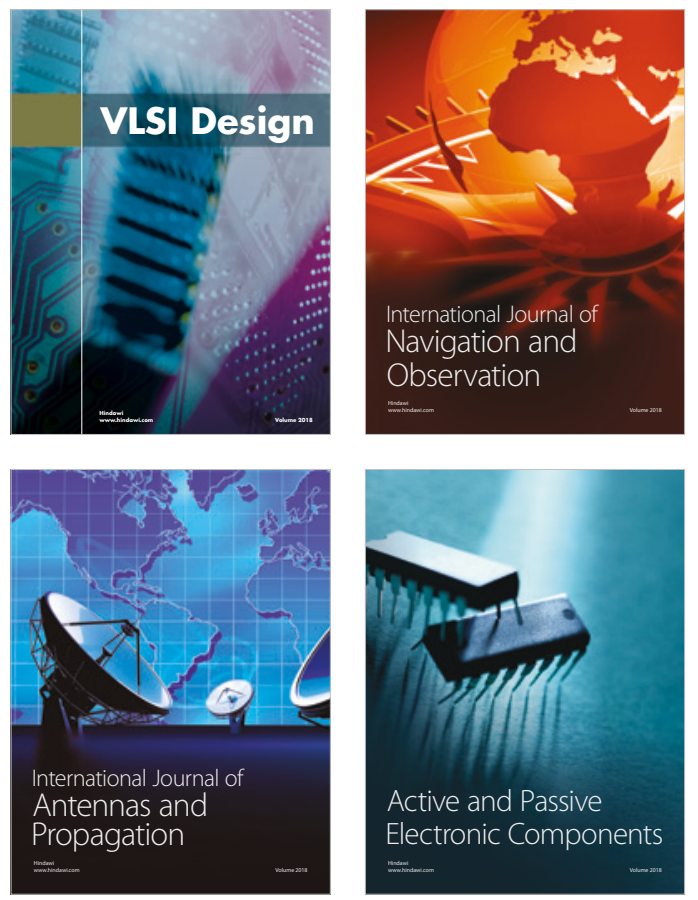
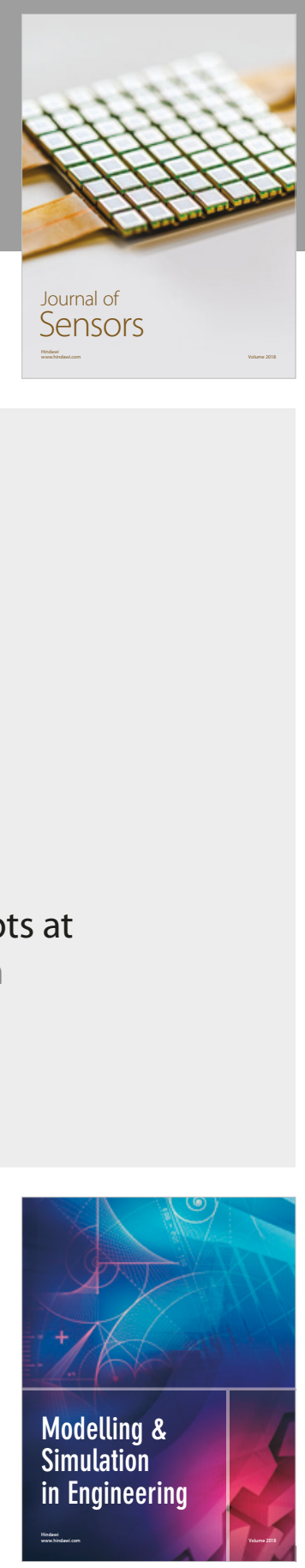

\section{Advances \\ Multimedia}
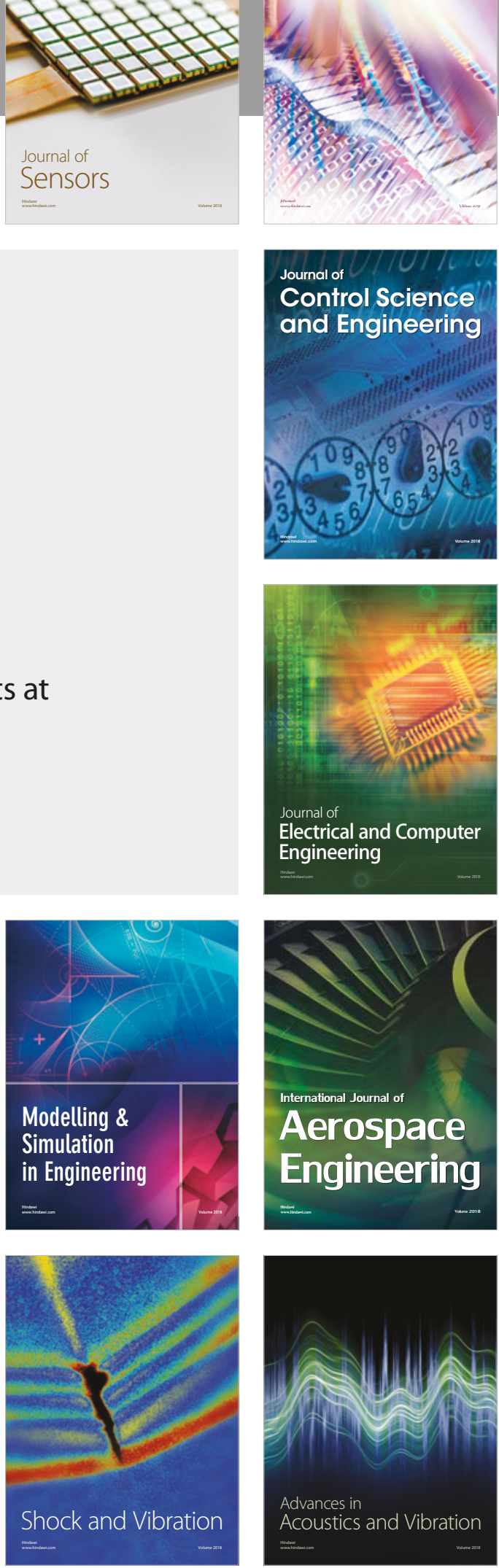